

\title{
Temperature Impact Analysis and Access Reliability Enhancement for 1T1MTJ STT-RAM
}

Bi Wu, Yuanqing Cheng, Jianlei Yang, Aida Todri-Sanial, Weisheng Zhao

\section{To cite this version:}

Bi Wu, Yuanqing Cheng, Jianlei Yang, Aida Todri-Sanial, Weisheng Zhao. Temperature Impact Analysis and Access Reliability Enhancement for 1T1MTJ STT-RAM. IEEE Transactions on Reliability, 2016, 65 (4), pp.1755-1768. 10.1109/TR.2016.2608910 . lirmm-01446148

\section{HAL Id: lirmm-01446148 \\ https://hal-lirmm.ccsd.cnrs.fr/lirmm-01446148}

Submitted on 25 Jan 2017

HAL is a multi-disciplinary open access archive for the deposit and dissemination of scientific research documents, whether they are published or not. The documents may come from teaching and research institutions in France or abroad, or from public or private research centers.
L'archive ouverte pluridisciplinaire HAL, est destinée au dépôt et à la diffusion de documents scientifiques de niveau recherche, publiés ou non, émanant des établissements d'enseignement et de recherche français ou étrangers, des laboratoires publics ou privés. 


\title{
Temperature Impact Analysis and Access Reliability Enhancement for 1T1MTJ STT-RAM
}

\author{
Bi Wu, Student Member, IEEE, Yuanqing Cheng, Member, IEEE, Jianlei Yang, Member, IEEE, Aida \\ Todri-Sanial, Member, IEEE, and Weisheng Zhao, Senior Member, IEEE
}

\begin{abstract}
Spin-Transfer Torque Magnetic Random Access Memory (STT-RAM) is a promising and emerging technology due to its many advantageous features such as scalability, nonvolatility, density, endurance and fast access speed. However, the operation of STT-RAM is severely affected by environmental factors such as process variations and temperature. As the temperature rockets up in modern computing systems, it is highly desirable to understand thermal impact on STT-RAM operations and reliability. In this paper, a thermal-aware MTJ model, calibrated and validated by experimental measurements, is proposed as the basis for thoroughly thermal aware analysis of 1T1MTJ STT-RAM cell structure. Using this model, we investigate temperature effect on memory cell access behavior in terms of access latency, energy and reliability on $45 \mathrm{~nm}$ technology node. Thermal impact on more advanced $11 \mathrm{~nm}$ technology node is also evaluated in the paper. Additionally, we propose a thermal-aware design for STT-RAM sensing circuit using bodybiasing technique, which can enlarge read margin dramatically to enhance read reliability under temperature variations. Moreover, our proposed technique can suppress read disturbance effectively as well. Experimental results show that our proposed sensing circuit can enlarge read margin by $2.47 \mathrm{X}$ when reading ' 0 ' and $3.15 \mathrm{X}$ when reading ' 1 ', and reduce read disturbance error rate by $55.6 \%$ on average.
\end{abstract}

Index Terms-Reliability, STT-RAM, Body-biasing, Temperature, Thermal modeling

\begin{tabular}{|c|c|}
\hline$S T T-R A M$ & $\begin{array}{l}\text { Spin-Transfer Torque Random } \\
\text { Access Memory }\end{array}$ \\
\hline$M T J$ & Magnetic Tunnel Junction \\
\hline$T M R$ & Tunnel Magneto-resistive Ratio \\
\hline$J_{c}$ & MTJ switching current density \\
\hline$J_{c 0}$ & MTJ switching current density at $0 \mathrm{~K}$ \\
\hline$k_{B}$ & Boltzmann constant \\
\hline$T$ & Absolute temperature \\
\hline$E_{b}$ & Energy barrier height \\
\hline$\Delta$ & Thermal stability of MTJ \\
\hline$t_{P W}$ & Switching current pulse width \\
\hline$\tau_{0}$ & Inverse of attempt frequency \\
\hline$V$ & Free layer volume \\
\hline$H_{K}$ & Effective anisotropy field \\
\hline
\end{tabular}

Bi Wu and Yuanqing Cheng have equal contributions in this work. Weisheng Zhao is the corresponding author.

$\mathrm{Bi} \mathrm{Wu}$, Yuanqing Cheng and Weisheng Zhao are with the School of Electrical and Information Engineering, Beihang University, Beijing, 100191 China (e-mail: bi.wu@buaa.edu.cn; yuanqing@ieee.org; weisheng.zhao@buaa.edu.cn).

Jianlei Yang is with the School of Computer Science and Engineering, Beihang University, 100191 China (e-mail: jerryyangs@gmail.com)

Aida Todri-Sanial is with LIRMM Laboratory, CNRS/University of Montpellier, Montpellier, 34095 France (e-mail: aida.todri@lirmm.fr).

This work is supported in part by China NSFC No. 61401008, Beijing NSF No. 4154076, and EU H2020 CONNECT grant agreement No. 688612

$\begin{array}{ll}\psi & \begin{array}{l}\text { Magnetization angle difference } \\ \text { between free layer and pinned layer } \\ \text { Magnetization angle between } \\ \text { free (pined) layer with Z axis } \\ \text { Magnetization angle between } \\ \text { free (pinned) layer with X axis } \\ \psi\end{array} \\ \text { MTJ width } \\ W & \text { MTJ length } \\ L & \text { Magnetic anisotropy } \\ K_{u} & \text { Magnetic damping constant } \\ \alpha & \text { Saturation magnetization } \\ M_{s} & \text { Oxide barrier thickness } \\ t_{o x} & \text { Free layer thickness } \\ t_{F} & \text { Gyromagnetic ratio } \\ \gamma & \text { Transistor source-body voltage } \\ V_{s b} & \text { Transistor threshold voltage } \\ V_{t h 0} & \text { when } V_{s b}=0 \\ \gamma & \text { Body effect coefficient } \\ \Phi_{F} & \text { Surface potential } \\ T_{r} & \text { Room temperature }\end{array}$

\section{INTRODUCTION}

$\mathbf{T}$ ECHNOLOGY node aggressive shrinking has made static power consumption a severe challenge to CMOS circuit due to sub-threshold leakage [1]. Memory systems including on-chip cache and off-chip main memory suffer from leakage power as their capacities get larger to meet ever-increasing memory bandwidth requirements. Even worse, leakage power consumption has gradually dominated total power budget [2]. To solve the elevated "power wall" problem [3], emerging non-volatile memory (NVM) technologies are proposed for ultra low power memory design. Among them, STT-RAM is one of the most promising candidates to replace conventional SRAM and DRAM due to its fast access speed comparable with SRAM, easy integration with CMOS process, and non-volatility [4]-[10].

Although STT-RAM has many advantages over traditional memory technologies such as SRAM and DRAM, it also faces many challenges hindering the widespread use in commercial market. Among them, read/write reliability is a big concern [11], [12]. As TMR usually lies between $100 \%$ and $200 \%$ [13], the resistance difference between $R_{a p}$ and $R_{p}$ is small and it is difficult to sense the resistance difference reliably. The transistor in series with MTJ in 1T1MTJ STT-RAM cell makes this issue even worse due to its non-negligible resistance. The main sources affecting STT-RAM reliability 
come from both process variations and environmental factors, such as temperature, magnetic field, etc.

Compared to extensive literatures investigating process variation effects on STT-RAM read/write reliability [14]-[16], temperature impact on STT-RAM read/write operations, however, lack of in-depth study. Although some papers investigated thermal impact on MTJ theoretically or experimentally [17] [19], the lack of thermal model for electrical simulation prevents the evaluation of thermal impact on circuit and architecture level design.

In the paper, we build a thermal-aware MTJ model for electrical simulation, which can capture TMR and switching current variations of MTJ with temperature fluctuations. After that, we use the MTJ model to investigate thermal impact on STT-RAM cell access behavior and its thermal sensitivity. The main contributions of this work are listed as follows:

- We develop a thermal-aware MTJ model for electrical simulation and the model is calibrated and validated against measurements from real STT-RAM prototypes.

- We thoroughly investigate temperature impact on access behavior of the typical 1T1MTJ cell structure from latency, energy and reliability perspectives. The temperature effect with technology scaling is also extensively investigated and some important observations and design inspirations are presented to assist future STT-RAM chip design.

- In order to improve read reliability of STT-RAM cell, we propose a body-biased sensing scheme that can enlarge read margin and suppress read disturbance effectively with only negligible area overhead.

The rest of paper is organized as follows. Section II presents preliminaries of STT-RAM and MTJ temperature dependency, which motivates our work. Section III illustrates our thermal model development for hybrid CMOS/MTJ electrical simulation. Section IV analyzes temperature effect on 1T1MTJ cell access behavior in $45 \mathrm{~nm}$ technology node. We also evaluate thermal impact on the more advanced $11 \mathrm{~nm}$ technology node to predict thermal scalability of STT-RAM in this section. Based on these analyses, we propose a sensing circuit adopting body-biasing technique to improve read margin and suppress read disturbance at different temperatures in Section V. Experimental results are shown in this section as well. Section VI presents the related work, and Section VII concludes the paper.

\section{Preliminaries of STT-RAM And Thermal DEPENDENCY OF MTJ DEVICE}

\section{A. Introduction to STT-RAM}

STT-RAM uses MTJ as data storage device depending on MTJ resistance. It is mainly composed of three layers, i.e., pinned layer, insulating barrier, and free layer. The nanopillar resistance $\left(R_{p}, R_{a p}\right)$ depends on the corresponding state of the magnetization of the two ferromagnetic layers Parallel( $\mathrm{P}$, denoted as data ' 0 ') or Anti-Parallel(AP, denoted as data ' 1 '). The resistance difference is characterized by $\mathrm{TMR}=\left(R_{a p}-R_{p}\right) / R_{p}$.

According to the magnetizations of free layer and pinned layer, MTJ can be classified into in-plane and perpendicular



(a)


(b)
Fig. 1: Illustration of MTJ structure. Data can be stored in the cell by switching magnetization of free layer to be parallel or anti-parallel to pinned layer. (a) In-plane MTJ: magnetizations of free and pinned layers are parallel to MTJ pillar surface. (b) Perpendicular MTJ: magnetizations of free and pinned layers are perpendicular to MTJ pillar surface.



Fig. 2: 1T1MTJ cell structure consisting of one transistor and one MTJ.

ones. The former MTJ, which is shown in Fig. 1(a), retains data by shape anisotropy and requires large current density to switch MTJ state. Compared to its in-plane counterpart, free layer magnetization of the latter one is perpendicular to MTJ pillar surface and requires much lower switching current density thus having better scalability than its in-plane counterpart [20]. Fig. 1(b) shows perpendicular MTJ and its two different states. In this article, we use in-plane MTJ technology in $45 \mathrm{~nm}$ technology node [21] and perpendicular MTJ technology in $11 \mathrm{~nm}$ technology node [22] for investigation.

The typical STT-RAM cell consists of 1 transistor and 1 MTJ called 1T1MTJ structure as shown in Fig. 2. It is widely used to construct STT-RAM memory array due to its high integration density and simplicity for fabrication [23], [24]. To read the cell, word line is enabled, and a read voltage can be applied between bit line and source line. The resulting sensing current will be compared to that of reference cell to distinguish ' 0 ' from ' 1 ' being read out. The reference cell consists of two MTJs, one in parallel state and the other in anti-parallel state. To write data into memory cell, word line is enabled and a voltage is applied between bit line and source line to generate sufficient switching current. Depending on polarity of writing current, a ' 0 ' or ' 1 ' can be written. Next, we will analyze temperature impact on STT-RAM cell, which motivates our work. 




Fig. 3: TMR and switching current variations at different temperatures [21].

\section{B. Thermal dependency of 1T1MTJ STT-RAM cell}

As computing enters into multi-core and many core era, power density on chip increases sharply and makes thermal issue a big problem of modern processors [25]. STT-RAM integrated as on chip cache, which is one of its most possible applications, suffers from high temperature as well. In fact, as the core component of STT-RAM, MTJ itself is highly temperature dependent. Fig. 3 shows TMR dependence on temperature measured from a STT-RAM prototype [21]. The research from Qualcomm also got similar results [17].

As shown in the figure, TMR decreases with increased temperature. Since TMR has dominant impact on STT-RAM cell sensing reliability, it is imperative to consider temperature when investigating STT-RAM cell access behavior. On the other hand, Fig. 3 also indicates that MTJ switching threshold current decreases with temperature. It is because $E_{b}$ decreases with temperature as observed in [17], and MTJ switching current density depends on $E_{b}$ following the formula shown below,

$$
J_{c}=J_{c 0}\left[1-\frac{k_{b} T}{E_{b}} \ln \left(\frac{t_{P W}}{\tau_{0}}\right)\right]
$$

It indicates that switching current decreases with $E_{b}$. From Fig. 3, we can observe the switching current variation is coincide with the formula prediction. The reduced MTJ switching current is due to degraded MTJ's thermal stability in high temperature. It can be calculated by the following formula [26],

$$
\Delta=\frac{E}{k_{B} T}=\frac{H_{K} M_{S}}{k_{B} T} V
$$

From (2) we can observe that thermal stability decreases with temperature increases. In other words, it requires less current to switch MTJ state as temperature increases. On the balance, both TMR and switching current, which determine access behavior of MTJ, are affected by temperature variations. The thermal impact on transistor has been well studied. When temperature increases, carrier mobility degrades accordingly as shown in the following expression, $\mu$ is the carrier mobility of transistor, and depends on the temperature indicated by the following relation:

$$
\mu(T)=\mu\left(T_{r}\right)\left(\frac{T}{T_{r}}\right)^{-k_{u}}
$$

Therefore, the driving ability of transistor is affected by temperature as well. In summary, it is necessary to consider both MTJ and transistor thermal dependency when analyzing the access behavior of STT-RAM cell at different temperatures.

\section{MTJ Modeling TeChNique Considering TEMPERATURE EFFECT FOR HYBRID CMOS/MTJ ELECTRICAL SIMULATION}

Although there are several MTJ models available for circuit level simulation, such as [27]-[29], they can not capture temperature effects on several MTJ properties simultaneously or support direct hybrid CMOS/MTJ electrical simulation. To facilitate thermal analysis of STT-RAM cell at circuit level and above, we develop a thermal model of MTJ based on MTJ model proposed by [30] and fit our model through measured data from a STT-RAM prototype [21].

Firstly, in order to capture temperature effect on TMR, we use the following approximation formula to calculate TMR at zero biasing voltage, i.e., $T M R(0)$,

$$
T M R(0)=a_{1}+a_{2} \cos \left(T \times b_{2}\right)+b_{1} \sin \left(T \times b_{2}\right)
$$

where $a_{1}, a_{2}, b_{1}, b_{2}$ are fitting parameters to match measurements from [17].

Note that TMR depends not only on temperature but also on bias voltage as in [31]. We use the following formula to calculate TMR under different biasing voltages:

$$
\operatorname{TMR}\left(V_{\text {bias }}\right)=\frac{T M R(0)}{1+V_{\text {bias }}^{2} / V_{h}^{2}}
$$

Therefore, TMR can be modeled as a function of both temperature and biasing voltage. MTJ thermal stability $\Delta$ depends on $M_{s}$ as shown below,

$$
\Delta=\frac{M_{s} H_{K} V \cos ^{2}(\theta)}{k_{B} T}
$$

Note that $M_{s}$ is temperature dependent as well according to [32]. We use the following formula to define $M_{s}$ such that thermal stability values at different temperatures fit with measurement values from [17]:

$$
M_{s}=p_{1} T^{3}+p_{2} T^{2}+p_{3} T+p_{4}
$$

where $p_{1}, p_{2}, p_{3}$ and $p_{4}$ are fitting parameters. Based on (6) and (7), we can obtain thermal stability at different temperatures.

After that, we use the work flow similar to that proposed in [30] to integrate temperature effect in MTJ model as shown in Fig. 4. The inputs of our model include MTJ size, barrier thickness, temperature, initial magnetization angles of free layer $(\theta$ and $\phi)$. As shown in the work flow, the temperature and bias voltage dependent TMR can be derived by (4) and (5) while temperature dependent thermal stability can be calculated from (6) and (7). Then, we can derive the MTJ switching current and spin torque induced by the injected spin polarized current. The spin torque together with uniaxial anisotropy torque, and shape anisotropy are fed into RHS of LLG equation [33] to update magnetization angle of free layer, i.e., $\theta$ and $\phi$, until MTJ approaches a steady state (anti-parallel state or parallel state). Since both in-plane and perpendicular 


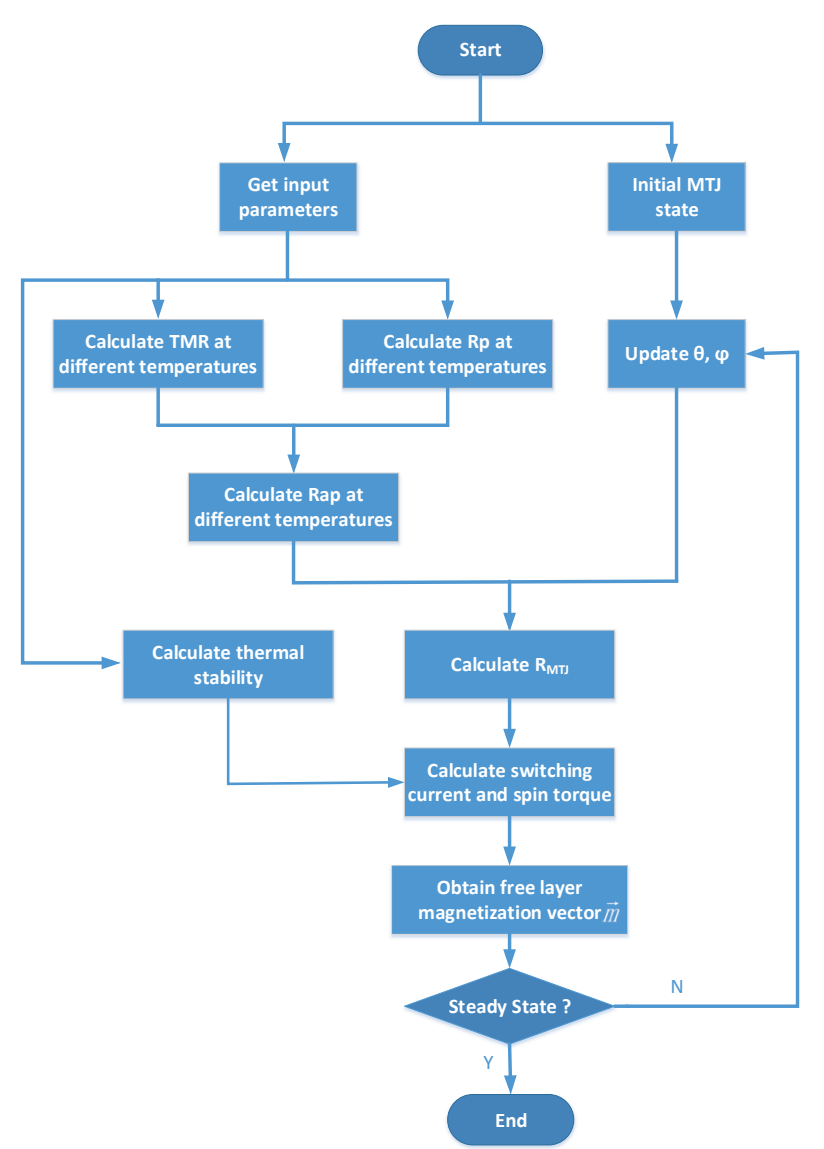

Fig. 4: The work flow of our thermal model

MTJ switching procedures are governed by LLG equation, our thermal model can be applied to both cases. The thermal model is implemented in Verilog-AMS and can be used for electrical simulation. Fig. 5 compares $R_{p}, R_{a p}$ and switching current derived by our model to measurements from a prototype chip [21] in different biasing voltages and temperatures. It shows that our thermal model can capture TMR and switching current variations accurately at different temperatures. The modeling errors of $R_{p}, R_{a p}$ and switching currents under different temperatures are within $10 \%$ of experimental meansurements. In next section, we will use this model to analyze temperature impact on read/write operations of a typical 1T1MTJ STTRAM cell.

\section{Temperature IMPACT ON 1T1MTJ STT-RAM CELL OPERATIONS}

\section{A. Introduction to Read/Write Circuit of ITIMTJ STT-RAM Cell}

We adopt the writing circuit proposed by [31] to perfor$\mathrm{m}$ write operation of 1T1MTJ STT-RAM cell. The circuit schematic is drawn in Fig. 6. By enabling transistor pair in diagonal, we can inject current in different directions, and write data ' 0 ' or ' 1 ' into the cell accordingly. For example, transistors $\mathrm{P} 0$ and $\mathrm{N} 1$ are enabled when writing ' 0 ' since the injected current flowing from upper left $V_{d d}$ to lower right ground can switch MTJ from anti-parallel state to parallel

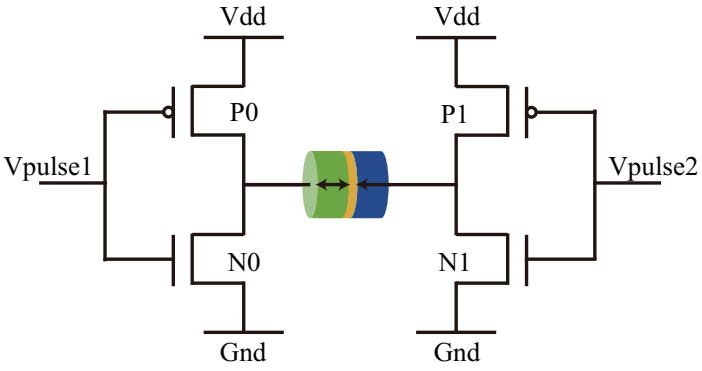

Fig. 6: Write circuit schematic of 1T1MTJ cell.

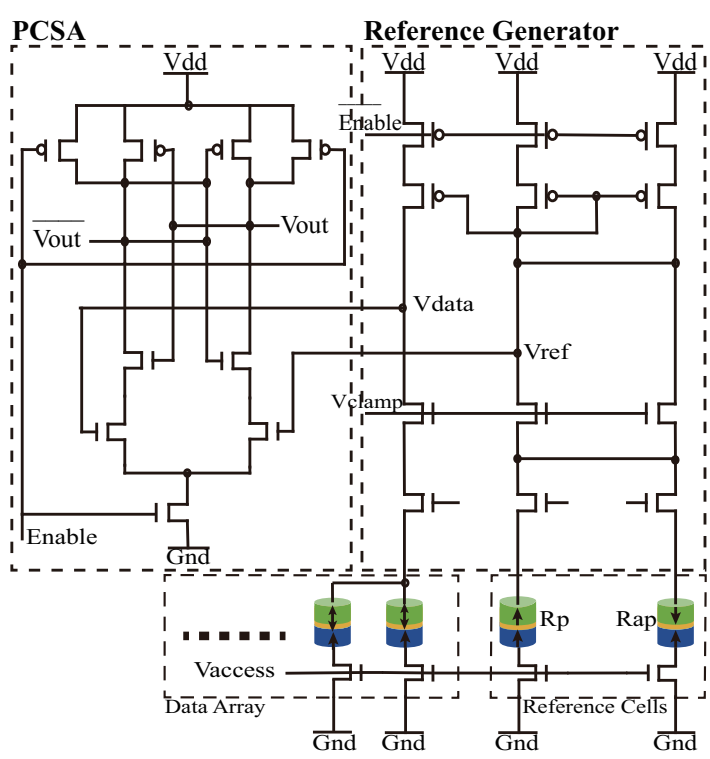

Fig. 7: Read circuit schematic of 1T1MTJ cell.

TABLE I: MTJ parameters used in our simulations

\begin{tabular}{|l|c|}
\hline Symbol & Value \\
\hline$L$ & $40 \mathrm{~nm}$ \\
$W$ & $100 \mathrm{~nm}$ \\
$K_{u}$ & $5 \times 10^{5} \mathrm{~A} / \mathrm{m}$ \\
$\alpha$ & 0.03 \\
$M_{s}$ at $25^{\circ} \mathrm{C}$ & $3.68 \times 10^{3} \mathrm{~A} / \mathrm{m}$ \\
$t_{o x}$ & $0.85 \mathrm{~nm}$ \\
$t_{F}$ & $33.55 \mathrm{~nm}$ \\
$\gamma$ & $1.76 \times 10^{7} \mathrm{rad} /(s \cdot T)$ \\
\hline
\end{tabular}

state. To write ' 1 ', we need to enable P1 and N0 to reverse switching current direction.

Considering read operations, we adopt the widely used twostage sensing scheme proposed by [8] and [34] as shown in Fig. 7. The first stage shown on the right part of the figure compares sensing current of data cell with that of reference cell. A clamping voltage is applied on both data cell and reference cell to make sure both sensing paths have the same voltage. Then, the sensing current difference between data cell path and reference path is converted to voltage difference via uploading transistors. Since the sensing voltage difference is usually only tens of millivolts being too small to be recognized by the following logic stage, it needs to be amplified by the second stage to generate full-swing signals, which is shown 


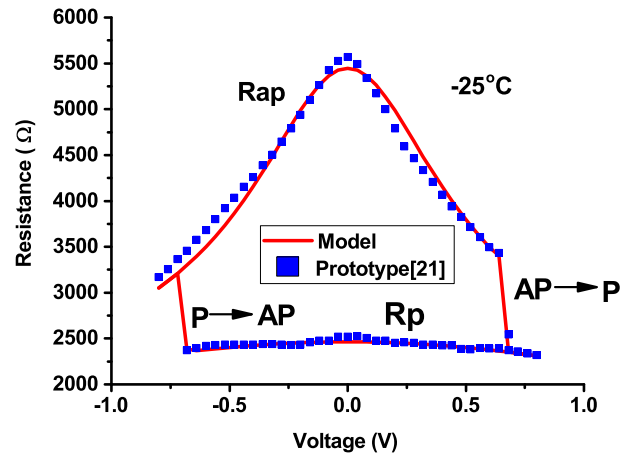

(a)

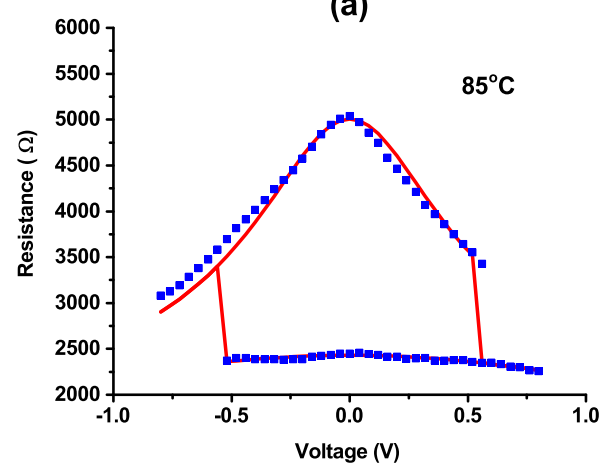

(c)

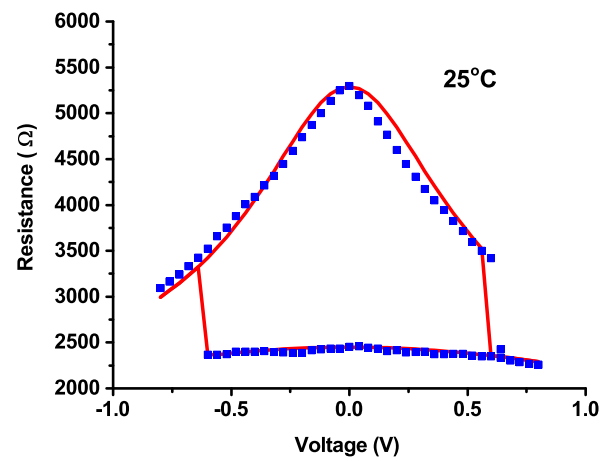

(b)



(d)

Fig. 5: Validation of our thermal-aware MTJ model against measurements from [21] (a) comparison of $-25^{\circ} \mathrm{C}$ case. (b) comparison of $25^{\circ} \mathrm{C}$ case. (c) comparison of $85^{\circ} \mathrm{C}$ case. (d) comparison of $125^{\circ} \mathrm{C}$ case.

on the left part of Fig. 7. In the following subsections, we use 45nm PTM CMOS model [35] and MTJ thermal model developed above to analyze temperature impact on reading and writing behaviors of STT-RAM cell. The parameters of MTJ model used for evaluation are listed in Table I.

\section{B. Temperature Effect on Reading Operations}

In the following, we evaluate temperature effect on STTRAM cell's reading behavior in terms of performance, energy and reliability using Cadence Spectre [36].

Fig. 8 plots sensing amplifier output voltage waveforms at four different temperatures (i.e., $-25^{\circ} \mathrm{C}, 25^{\circ} \mathrm{C}, 85^{\circ} \mathrm{C}$ and $\left.125^{\circ} \mathrm{C}\right)$. The read pulse applied between bit line and source line has 2 ns width to make sure data can be sensed reliably. Fig. 8a shows output waveforms of the second stage of sensing amplifier when ' 0 ' is read. Fig. 8 b shows output waveforms when read ' 1 ' at different temperatures. Considering reading ' 0 ', read latency increases with temperature. For instance, it is $510 \mathrm{ps}$ at $-25^{\circ} \mathrm{C}$ while it becomes $1.06 \mathrm{~ns}$ at $125^{\circ} \mathrm{C}$. Reading ' 1 ' operation indicates the similar trend. By examining sensing signals, we observe that read latency increase is mainly due to two factors. First of all, read margin shrinks with temperature (showed in Table II). Read margin is defined as the difference between reading signal and reference signal. Refer to Fig. 5, $R_{p}$ of MTJ remains almost the same at different temperatures while $R_{a p}$ becomes smaller at higher temperature. The data sensing voltage output from the first stage of sensing circuit can be expressed as:

$$
V_{d a t a}=V_{d d}-\frac{V_{c l a m p}}{R_{m t j}} \times R_{l o a d}
$$

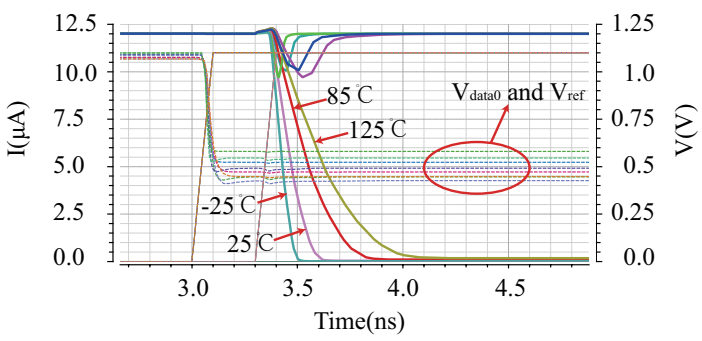

(a) Read '0' latencies at four temperatures.



(b) Read ' 1 ' latencies at four different temperatures.

Fig. 8: Read latencies of 1T1MTJ cell at different temperatures

Reference sensing voltage output is,

$$
V_{\text {ref }}=V_{d d}-\left(\frac{V_{\text {clamp }}}{R_{a p}}+\frac{V_{\text {clamp }}}{R_{p}}\right) / 2 \times R_{\text {load }}
$$

where $V_{\text {clamp }}$ is clamping voltage applied over data cell and reference cell. $R_{\text {load }}$ is resistance of uploading transistor. When sensing ' 0 ', $R_{m t j}=R_{p}$, and read margin $\left|V_{\text {data }}-V_{\text {ref }}\right|=V_{\text {clamp }} R_{\text {load }}\left(\frac{1}{2 R_{p}}-\frac{1}{2 R_{a p}}\right)$. As shown in Fig. 
TABLE II: Read margins and energy consumptions at different temperatures at $45 \mathrm{~nm}$ technology node.

\begin{tabular}{|c|c|c|c|c|}
\hline & $-25^{\circ} \mathrm{C}$ & $25^{\circ} \mathrm{C}$ & $85^{\circ} \mathrm{C}$ & $125^{\circ} \mathrm{C}$ \\
\hline \multicolumn{5}{|c|}{ Read margins at different temperatures. (unit: $\mathrm{mV}$} \\
\hline Read '0' & 35.0 & 32.3 & 26.3 & 22.1 \\
\hline Read '1' & 40.3 & 32.0 & 20.2 & 14 \\
\hline \multicolumn{5}{|c|}{ Read energy at different temperatures. (unit: fJ) } \\
\hline Read '0' & 18.4 & 18.9 & 19.7 & 21.1 \\
\hline Read '1' & 17.7 & 18.5 & 19.5 & 19.9 \\
\hline
\end{tabular}

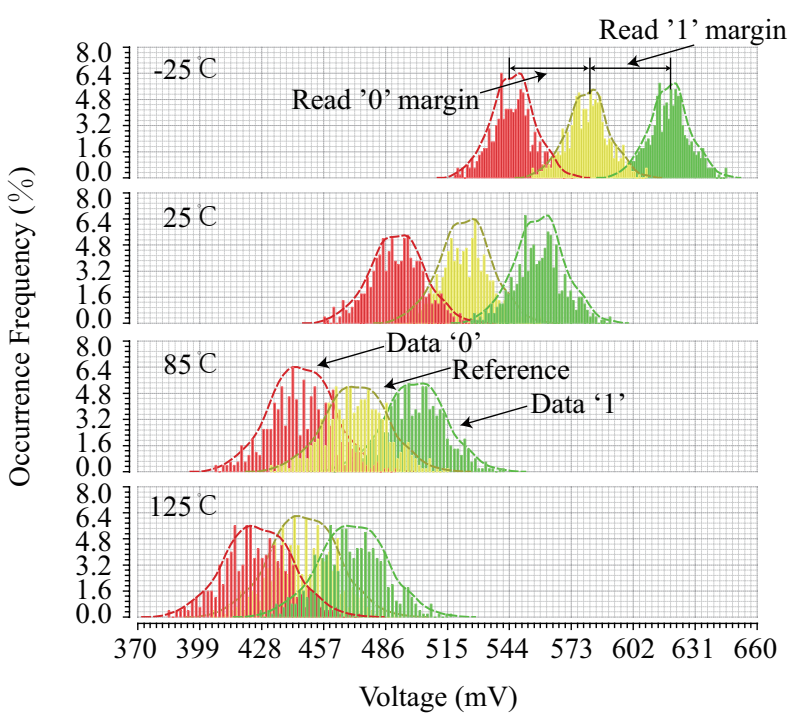

Fig. 9: Sensing and reference voltage distributions at different temperatures through 1000 Monte Carlo simulations.

$5, R_{a p}$ decreases with temperature while $R_{p}$ remains almost unchanged. Therefore, read margin is smaller in higher temperature making sensing time much longer. Likewise, when reading ' 1 ', sensing margin also becomes smaller in higher temperature. Hence, sensing latency increases with temperature. Another reason of read latency increasing with temperature is that transistor driving ability also degrades with temperature. Since the second stage relies on discharging speed difference between two discharging paths, degrading current driving ability makes data sensing more difficult in higher temperature. Table II lists read margins at different temperatures when read ' 1 ' and ' 0 ' respectively, which validates our theoretical analysis. As shown in the table, when temperature increases from $-25^{\circ} \mathrm{C}$ to $125^{\circ} \mathrm{C}$, read margin of reading ' 1 ' decreases by $65.3 \%$, and that of reading ' 0 ' decreases by $36.9 \%$. Therefore, temperature has significant effect on read performance, and should be thoroughly considered during STT-RAM cell design such that it can achieve given performance specification across the whole working temperature range.

Read energy at different temperatures is also tabulated in Table. II, which is consistent with data in [37]. Read energy increases by $14.7 \%$ from $-25^{\circ} \mathrm{C}$ to $125^{\circ} \mathrm{C}$ when reading ' 0 '. Reading ' 1 ' shows similar energy increasing with temperature. Based on these results, we can observe that although driving current reduces as temperature, increased read latency has a larger impact on read energy consumption.

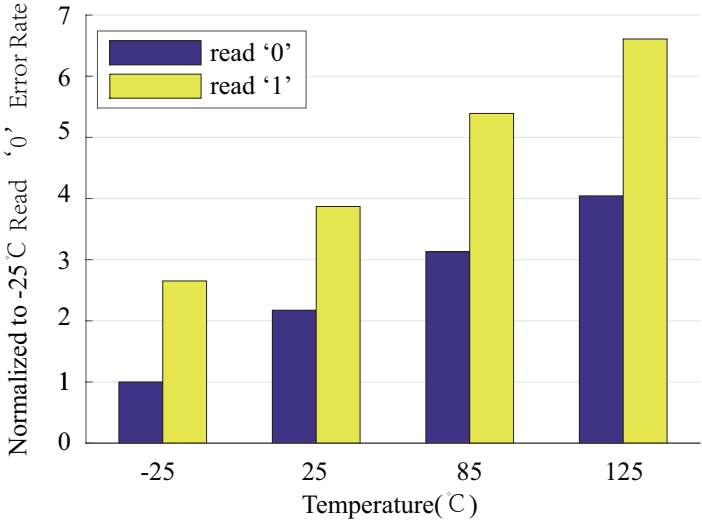

Fig. 10: Read error rate comparisons at different temperatures in $45 \mathrm{~nm}$ technology node. Both reading ' 0 ' and ' 1 ' error rates increase with temperature, and reading ' 1 ' error rate is higher due to $R_{a p}$ variation with temperature.

TABLE III: Monte Carlo simulation parameters

\begin{tabular}{|l|r|r|}
\hline Parameters & Mean & Std.Dev \\
\hline Channel Length & $45 \mathrm{~nm}$ & $4 \%$ \\
\hline Channel Width & $135 \mathrm{~nm}$ & $4 \%$ \\
\hline Threshold Voltage & $0.4 \mathrm{~V}$ & $16 \mathrm{mV}$ \\
\hline MTJ Width & $40 \mathrm{~nm}$ & $4 \%$ \\
\hline MTJ Length & $100 \mathrm{~nm}$ & $4 \%$ \\
\hline
\end{tabular}

Next, we evaluate read reliability of 1T1MTJ cell at different temperatures. To obtain sensing and reference voltage distributions, we perform 1000 Monte Carlo simulations for reading ' 0 ' and reading ' 1 ' respectively. The simulation settings are shown in Table.III. The process variations of transistor gate length, threshold voltage and MTJ size are assumed to obey Gaussian distributions, and $\sigma / \mu$ of each parameter distribution is assumed as $4 \%$. Sensing voltage distributions at different temperatures are plotted in Fig. 9. From the figure, we have two important observations. Firstly, as the temperature increases, overlapping of read voltage and reference voltage distributions becomes more significant, which is attributed to shrinking read margin. Overlapping area denotes where read error may occur. Secondly, the reference voltage drifts with temperature instead of a fixed value. Since resistances of uploading PMOS transistors increase with temperature while MTJ resistance remains almost the same (for $R_{p}$ ) or becomes smaller $\left(R_{a p}\right)$, voltage drop on uploading PMOS transistors becomes larger in higher temperature. Therefore, both reference and data sensing voltage reduce with temperature. Fig. 10 dictates read error rates at different temperatures normalized to that of $-25^{\circ} \mathrm{C}$. We can observe that read error rate increases with temperature, and error rate at $125^{\circ} \mathrm{C}$ increases by $4 \mathrm{X}$ compared to that of $-25^{\circ} \mathrm{C}$ when reading ' 0 ' and increases by $3.3 \mathrm{X}$ when reading ' 1 '. Note that $R_{a p}$ decreasing with temperature on both data sensing path and reference path makes sensing ' 1 ' more error-prone. In summary, shrinking read margin degrades sensing reliability of STT-RAM, i.e., given the same sensing period, it is expected to incur more read errors at higher temperature. Therefore, the sensing circuit design for STT-RAM should take temperature into account 


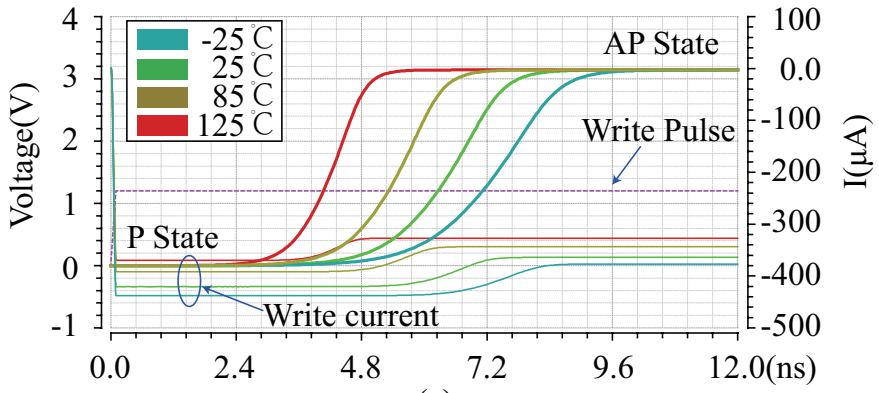

(a)

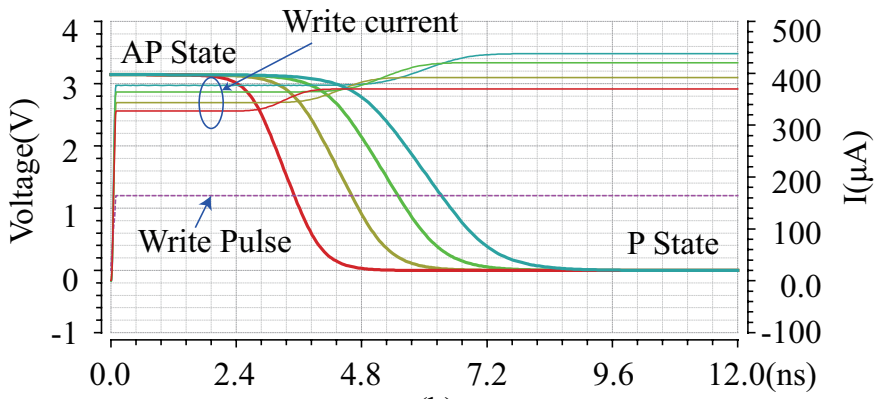

(b)

Fig. 11: Write currents at different temperatures. (a) write current decreases with temperature when MTJ is switched from $\mathrm{P}$ state to AP state due to reduced thermal stability. (b) write ' 0 ' current decreases with temperature as well due to the same reason.

TABLE IV: Write latencies and energy at different temperatures

\begin{tabular}{|c||c|c|c|c|}
\hline Write latency $(n s)$ & $-25^{\circ} \mathrm{C}$ & $25^{\circ} \mathrm{C}$ & $85^{\circ} \mathrm{C}$ & $125^{\circ} \mathrm{C}$ \\
\hline write '0' & 8.81 & 7.60 & 6.41 & 5.16 \\
\hline write '1' & 10.02 & 8.75 & 7.37 & 5.64 \\
\hline Write energy (pJ) & $-25^{\circ} \mathrm{C}$ & $25^{\circ} \mathrm{C}$ & $85^{\circ} \mathrm{C}$ & $125^{\circ} \mathrm{C}$ \\
\hline write '0' & 3.70 & 3.27 & 2.83 & 2.28 \\
\hline write '1' & 4.10 & 3.88 & 3.61 & 2.91 \\
\hline
\end{tabular}

to ensure it can work reliably across the whole working temperature range without violating reliability specification.

\section{Temperature Effect on Write Operations}

We use the write circuit shown in Fig. 6 in our simulation. Fig. 11 plots write current of writing ' 0 ' and ' 1 ' waveforms respectively at different temperatures. As shown in the figure, write current decreases with temperature for both writing ' 0 ' and ' 1 ' cases. For example, writing ' 1 ' current drops from $380 \mu \mathrm{A}$ in $-25^{\circ} \mathrm{C}$ to $330 \mu \mathrm{A}$ in $125^{\circ} \mathrm{C}$. As mentioned in Section II, MTJ's switching current depends on thermal stability which reduces with temperature. As a result, switching current decreases with temperature as well. Table IV lists write latencies and energy at different temperatures. We can observe that write latency decreases with temperature implying that MTJ can be switched more easily due to reduced thermal stability. As both write latency and current become smaller in higher temperature, MTJ switching energy also decreases with temperature as shown in Table IV. Note that writing ' 1 ' energy

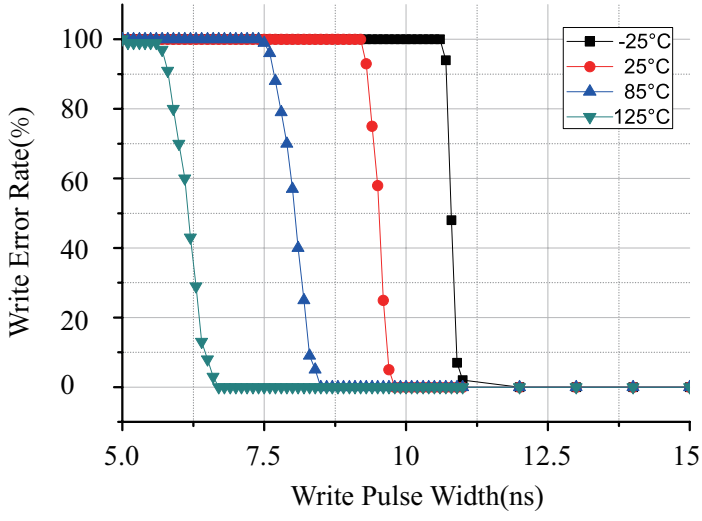

Fig. 12: Write error rate at different temperatures. Write latency decreases with temperature and write error rate curve shifts to the left with temperature increasing.

is higher than writing ' 0 ' energy since $\mathrm{P} \rightarrow \mathrm{AP}$ switching is more difficult than $\mathrm{AP} \rightarrow \mathrm{P}$ [21].

Next, we consider write error rate at different temperatures. The write pulse width is swept from 6ns to $11 \mathrm{~ns}$ which covers typical write latencies of STT-RAM. The simulation results are plotted in Fig. 12. We can observe that write error rate curve shifts to right remarkably as the temperature decreases. For instance, at $125^{\circ} \mathrm{C}, 6 \mathrm{~ns}$ is enough to ensure write error free while the write pulse width should be larger than $11 \mathrm{~ns}$ when temperature decreases to $-25^{\circ} \mathrm{C}$. Additionally, since write current lies in hundreds of $\mu A$ while read current is only tens of $\mu A$ in $45 \mathrm{~nm}$ technology node, read disturbance can be negligible ${ }^{1}$. Write errors mostly come from insufficient write pulse width.

In summary, high temperature incurs high read latency and energy. Read margin also shrinks with temperature making read reliability a challenge in high temperature. Whereas, write pulse can be shortened in high temperature and less errorprone. Additionally, due to large difference between write current and read current, read disturbance is not significant at $45 \mathrm{~nm}$ technology node. The write error is mainly caused by insufficient write pulse width or magnitude.

\section{Temperature impact on STT-RAM technology scalability}

As technology node shrinks, difference between write current and read current becomes smaller and read reliability will be a severe challenge for reliable operation of STT-RAM [11]. In this subsection, we will evaluate temperature impact on STT-RAM access operations at $11 \mathrm{~nm}$ technology node. We calibrate our MTJ model with measured data from [22] to match switching current, energy barrier, TMR and MTJ resistance of $11 \mathrm{~nm}$ MTJ at room temperature. Due to the lack of published measurements of MTJ parameters at different temperatures, we assume TMR and resistance variation trend with temperature at $11 \mathrm{~nm}$ technology node are similar to those of $45 \mathrm{~nm}$ technology node due to their similar material and stacking structure. Then, we can scale our thermal model to

\footnotetext{
${ }^{1}$ Read disturbance means erronously switching of MTJ during read procedure due to large read current.
} 


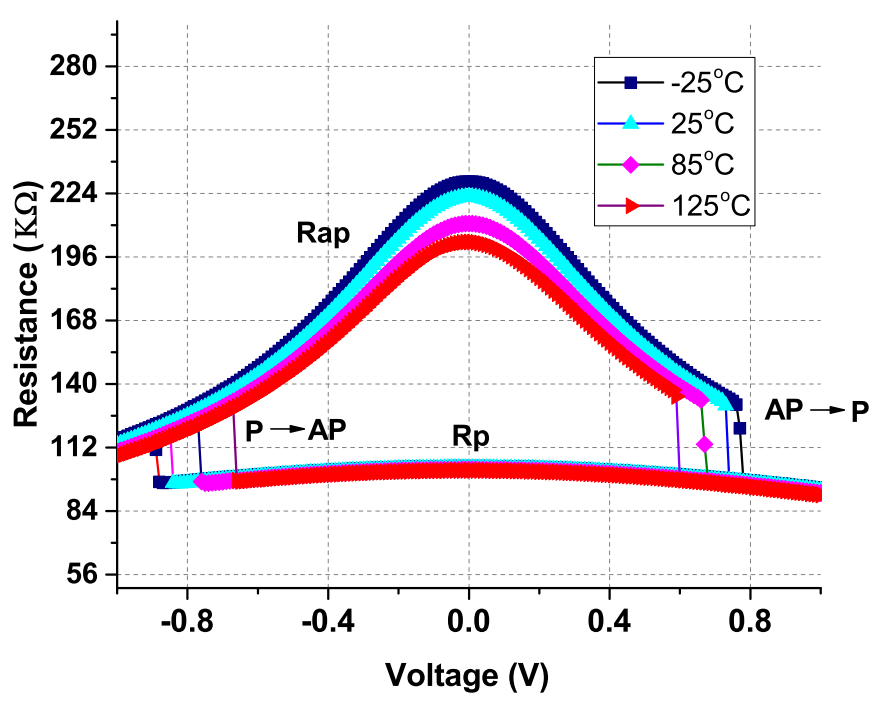

Fig. 13: Temperature effect on switching current and MTJ resistance at $11 \mathrm{~nm}$ technology node. Switching current, TMR and MTJ resistance at room temperature are calibrated with measurements presented in [22].

$11 \mathrm{~nm}$ accordingly. The relationship of MTJ resistance and biasing voltage at different temperatures derived from HSPICE simuations are plotted in Fig. 13. The $\mathrm{X}$ axis represents MTJ biasing voltage in Volt, and the $\mathrm{Y}$ axis is resistance of MTJ in $K \Omega$. As shown in Fig. 13, Rp and Rap derived by our thermal model are $224 K \Omega$ and $103 K \Omega$ respectively. The maximum error compared to data from [22] is $3 \%$. The switching current from [22] at $11 \mathrm{~nm}$ technology node is below $10 \mu \mathrm{A}$ while that derived from our thermal model is about $7 \mu \mathrm{A}$ which matches with experimental result as well. Therefore, our scaled thermal model can be used to predict $1 \mathrm{X} \mathrm{nm}$ STT-RAM thermal behaviors accurately.

From the figure, we can observe several interesting points. First, both $R_{p}$ and $R_{a p}$ are much larger than those of $45 \mathrm{n}$ $\mathrm{m}$ MTJ. The reason is that it is preferable to maintain a roughly constant RA (the product of MTJ resistance and area) across different generations of MTJ fabrication technology. The MTJ area shrinks from $40 \mathrm{~nm} \times 100 \mathrm{~nm}$ to $\pi \times 5.5 \mathrm{~nm}^{2}$, which makes MTJ resistance larger to maintain RA value unchanged. Secondly, although $11 \mathrm{~nm}$ MTJ switching current is much smaller than that of 40nm MTJ, considering sharply increase of MTJ resistance, the supply voltage of $1 \mathrm{X} \mathrm{nm}$ CMOS technology may be not enough to provide enough switching current for MTJ switching. Therefore, we have to apply a higher voltage in $11 \mathrm{~nm}$ technology node to write MTJ successfully. New material or novel MTJ structure should be explored to reduce MTJ switching current further for compatible hybrid CMOS/Magnetic 1T1MTJ cell design at more advanced technology node as mentioned in [22]. However, this topic is out of the scope of this article and we will take this issue as our future work.

In the following, we adopt 16nm CMOS PTM model [35] and $11 \mathrm{~nm}$ MTJ for circuit simulations. Using the similar sensing circuit as that for $45 \mathrm{~nm}$ case, we evaluate temperature effect on read behavior of 1Xnm 1T1MTJ STT-RAM. The
TABLE V: Read performance and energy at different temperatures in $11 \mathrm{~nm}$ MTJ technology node

\begin{tabular}{|c||c|c|c|c|}
\hline Read latency (ps) & $-25^{\circ} \mathrm{C}$ & $25^{\circ} \mathrm{C}$ & $85^{\circ} \mathrm{C}$ & $125^{\circ} \mathrm{C}$ \\
\hline read '0' & 410 & 420 & 440 & 450 \\
\hline read '1' & 413 & 421 & 430 & 439 \\
\hline Read current $(\mu A)$ & $-25^{\circ} \mathrm{C}$ & $25^{\circ} \mathrm{C}$ & $85^{\circ} \mathrm{C}$ & $125^{\circ} \mathrm{C}$ \\
\hline read '0' & 2.76 & 2.46 & 2.13 & 1.93 \\
\hline read '1' & 1.88 & 1.73 & 1.59 & 1.49 \\
\hline Read energy (fJ) & $-25^{\circ} \mathrm{C}$ & $25^{\circ} \mathrm{C}$ & $85^{\circ} \mathrm{C}$ & $125^{\circ} \mathrm{C}$ \\
\hline read '0' & 3.92 & 3.47 & 3.08 & 2.87 \\
\hline read '1' & 3.61 & 3.18 & 2.76 & 2.56 \\
\hline
\end{tabular}

TABLE VI: Write latency and energy at different temperatures of $11 \mathrm{~nm} 1 \mathrm{~T} 1 \mathrm{MTJ}$ memory cell

\begin{tabular}{|c||c|c|c|c|}
\hline Write latency (ns) & $-25^{\circ} \mathrm{C}$ & $25^{\circ} \mathrm{C}$ & $85^{\circ} \mathrm{C}$ & $125^{\circ} \mathrm{C}$ \\
\hline write '0' & 7.86 & 6.69 & 5.38 & 3.98 \\
\hline write '1' & 9.09 & 7.68 & 6.3 & 4.65 \\
\hline Write energy (fJ) & $-25^{\circ} \mathrm{C}$ & $25^{\circ} \mathrm{C}$ & $85^{\circ} \mathrm{C}$ & $125^{\circ} \mathrm{C}$ \\
\hline write '0' & 81.7 & 68.3 & 53.0 & 37.9 \\
\hline write '1' & 102 & 84.6 & 66.6 & 47.3 \\
\hline
\end{tabular}

read latencies, currents and energy consumptions at different temperatures are listed in Table. V. From the table, we can observe that MTJ read latency becomes much smaller in $11 \mathrm{~nm}$ technology node compared to that of $45 \mathrm{~nm}$ case, which shows a good scalability of MTJ technology. The read latency of $45 \mathrm{~nm}$ MTJ is $1 \mathrm{~ns}$ at $125^{\circ} \mathrm{C}$ while it is about $0.4 \mathrm{~ns}$ for $11 \mathrm{~nm}$ MTJ. On the other hand, as temperature increases, read latency of $11 \mathrm{~nm}$ memory cell also increases accordingly because of shrinking read margin, which has the similar trend as that of $45 \mathrm{~nm}$ case. Considering read current, it is much smaller in $11 \mathrm{~nm}$ case, which only requires several $\mu A$ for reading. The read current decreases with temperature, which is similar as $45 \mathrm{~nm}$ case. It is caused by degraded driving ability of CMOS transistor at high temperature. However, reading energy decreases with temperature as shown in Table. V. It is because decreasing read current dominates the read energy in $11 \mathrm{~nm}$ technology node, while increasing read latency dominates the read energy in $45 \mathrm{~nm}$ technology node. In summary, compared to Table II, both read latency and energy reduce significantly at $11 \mathrm{~nm}$ node which indicates good scalability of MTJ with technology node shrinking.

Table VI tabulates write energy and latencies of $11 \mathrm{~nm}$ STTRAM cell working at different temperatures. From the table, we observe that write latencies become much smaller than those of $45 \mathrm{~nm}$ STT-RAM cell. Write energy also reduces compared to $45 \mathrm{~nm}$ case. The reduction of write energy and latency is attributed to reducing thermal stability of MTJ at $11 \mathrm{~nm}$ technology [22]. As shown in the table, write energy is reduced by two orders of magnitude compared to $45 \mathrm{~nm}$ case which indicates the good scalability of STT-RAM technology. As the temperature increases, both write latency and write energy decrease which are similar to $45 \mathrm{~nm}$ case because of reduced thermal stability in high temperature. Moreover, for $11 \mathrm{~nm}$ technology node, latencies of writing ' 1 ' are significantly larger than those when writing ' 0 '. It is because the switching asymmetry becomes more severe due to large difference between $R_{p}$ and $R_{a p}$ at $11 \mathrm{~nm}$ technology node. 


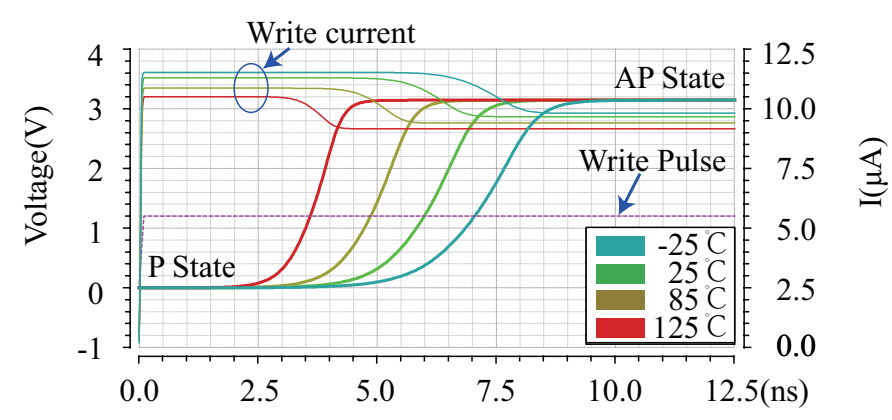

(a)

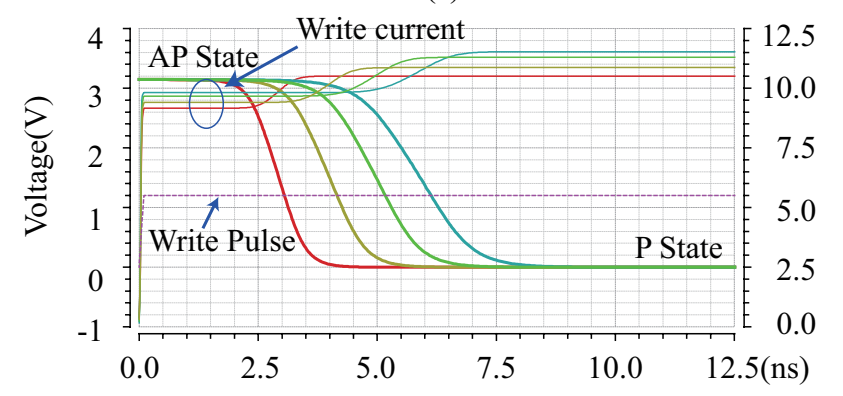

(b)

Fig. 14: Write current variations with temperature of $11 \mathrm{~nm}$ MTJ. (a) write ' 1 ' current decreases from $11.5 \mu \mathrm{A}$ to $10 \mu \mathrm{A}$ when temperature elevates from $-25^{\circ} \mathrm{C}$ to $125^{\circ} \mathrm{C}$. (b) write ' 0 ' current decreases from $10 \mu A$ to $9 \mu A$ when temperature elevates from $-25^{\circ} \mathrm{C}$ to $125^{\circ} \mathrm{C}$.

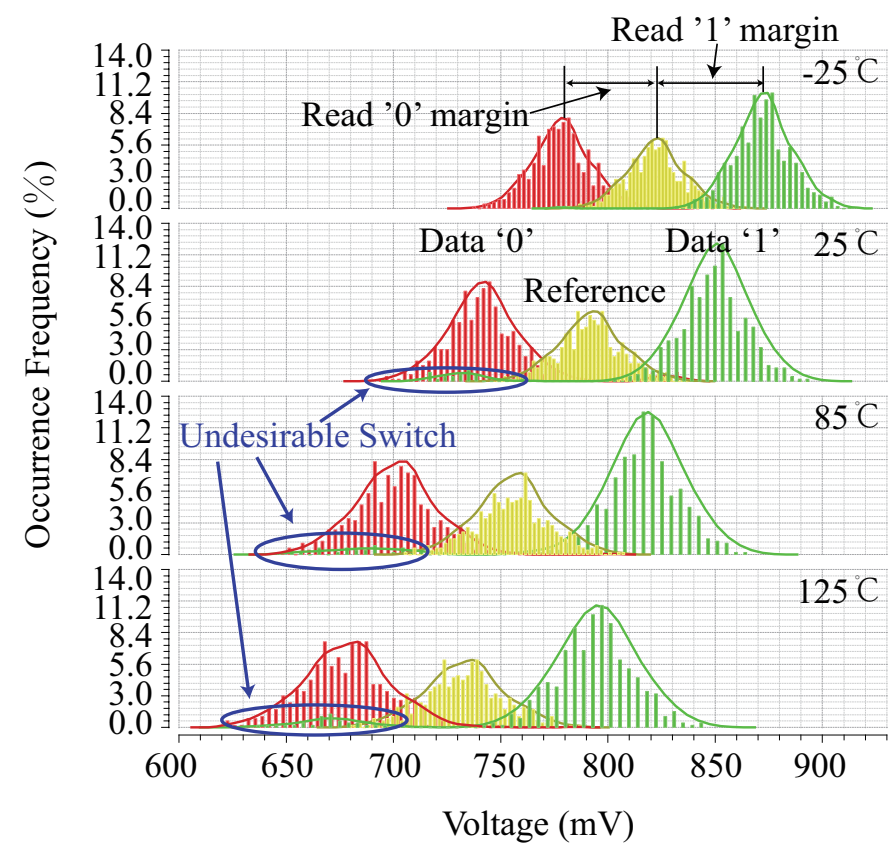

Fig. 15: Read voltage distributions of $11 \mathrm{~nm}$ STT-RAM cell at different temperatures. In addition to read margin shrinking with temperature similar to $45 \mathrm{~nm}$ case. When reading ' 0 ' from $11 \mathrm{~nm}$ MTJ, it indicates considerable read disturbance occurrences.

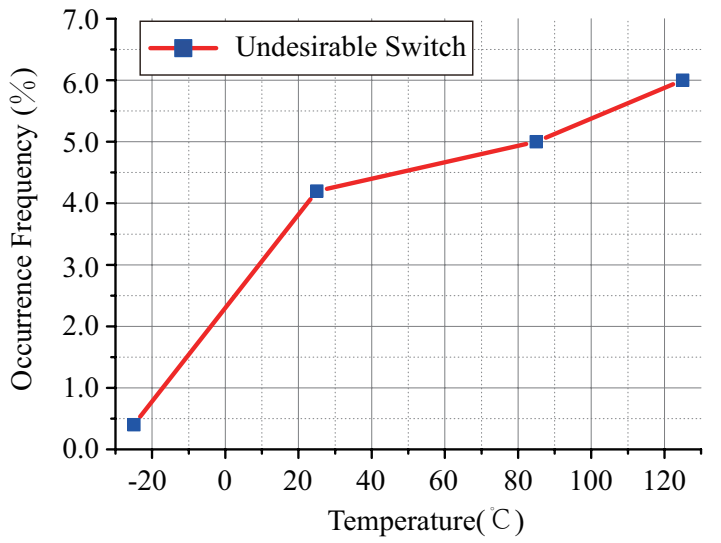

Fig. 16: Read error rate due to read disturbance at different temperatures.

The write currents at different temperatures are plotted in Fig. 14. As shown in the figure, write ' 1 ' current decreases from $11.5 \mu \mathrm{A}$ to $10 \mu \mathrm{A}$ when temperature elevates from $-25^{\circ} \mathrm{C}$ to $125^{\circ} \mathrm{C}$ while write ' 0 ' current decreases from $10 \mu \mathrm{A}$ to $9 \mu \mathrm{A}$ when temperature elevates from $-25^{\circ} \mathrm{C}$ to $125^{\circ} \mathrm{C}$. Additionally, at the same temperature, it requires higher switching current for $\mathrm{P} \rightarrow \mathrm{AP}$ switching. For instance, at $25^{\circ} \mathrm{C}$, the write current is about $9 \mu A$ to switch MTJ from AP state to P state while switching from $\mathrm{P}$ to $\mathrm{AP}$ needs $11 \mu A$ current.

Although the reducing switching current is helpful to reduce writing energy, it also introduces read disturbance in advanced technology node as read current approaches write current more closely. To evaluate read reliability, we perform 1000 Monte Carlo simulations at $-25^{\circ} \mathrm{C}, 25^{\circ} \mathrm{C}, 85^{\circ} \mathrm{C}$ and $125^{\circ} \mathrm{C}$ respectively. The $\sigma / \mu$ of CMOS transistor threshold voltage, gate length and MTJ radius distributions are assumed to be $4 \%$ in the simulation. Refer to read circuit used in the paper shown in Fig. 7, only ' 1 ' $\rightarrow$ ' 0 ' switching can occur during reading procedure. The sensing and reference voltage distributions are illustrated in Fig. 15. Except for read margin shrinking with temperature, we can observe read disturbance occurs during reading ' 1 ', which is circled in the figure. Compared to $45 \mathrm{~nm}$ case, the read disturbance is much more severe. To show it more clearly, we plot read error caused by read disturbance at different temperatures in Fig. 16. As shown in the figure, the read error rate caused by read disturbance increases from $0.5 \%$ to $6 \%$ when temperature increases from $-25^{\circ} \mathrm{C}$ to $125^{\circ} \mathrm{C}$ due to reduced thermal stability of MTJ at high temperature. Therefore, read disturbance will be a big concern when technology node shrinks below $1 \mathrm{X}$ nm regime, and has strong dependence on temperature.

\section{IMPROVING READING RELIABILITY OF STT-RAM WITH TEMPERATURE VARIATIONS BY BODY-BIASING TECHNIQUE}

From the above analyses, we find that read margin shrinks with temperature making sensing reliability a big challenge for STT-RAM especially at high temperature. Additionally, read disturbance aggravates as temperature increases as well. To 


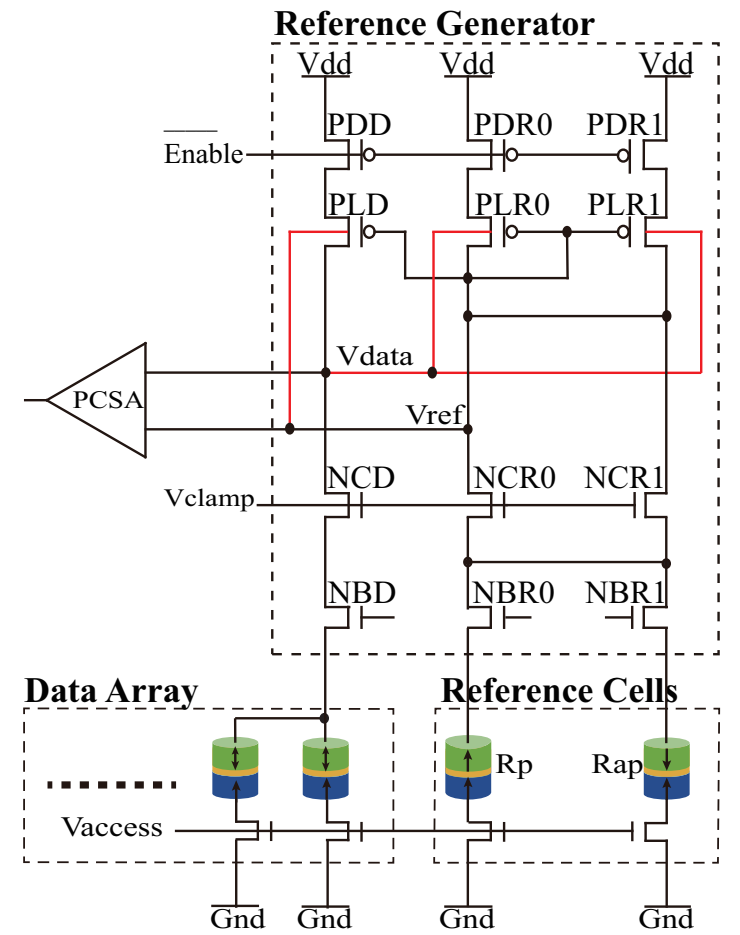

Fig. 17: Body biased sensing circuit schematic.

overcome these problems, we propose a body-biasing technique to adjust reference voltage dynamically such that read margin can be enlarged and read reliability can be improved. Moreover, if read margin remains unchanged, we can reduce the sensing current accordingly, and limit read disturbance at very deep sub-micron regime.

\section{A. The working principle of our proposed body biasing sens- ing circuit}

Based on the scheme we used for read operation evaluations above, which is proposed by [8], we propose a bodybiasing technique shown in Fig. 17 to enlarge read margin of 1T1MTJ STT-RAM cell. The main idea is that through adjusting body voltage of uploading PMOS transistors, their threshold voltages and driving abilities can be enhanced or suppressed dynamically to enlarge voltage difference between data sensing path and reference path. In the proposed read circuit, data output in the first sensing stage is connected to body terminals of two uploading PMOS transistors (PLR0 and PLR1) on reference branches while reference output is connected to the body terminal of uploading PMOS transistor on data sensing branch PLD) as shown in the figure. The working detail of body-biasing sensing circuit is analyzed as follows.

Considering body effect, the threshold voltage of PMOS transistor is given by the following equation,

$$
\left|V_{t h}\right|=\left|V_{t h 0}\right|+|\gamma|\left(\sqrt{\left|2 \Phi_{F}+V_{S B}\right|}-\sqrt{\mid 2 \Phi_{F \mid}}\right)
$$

As the body voltage changes, the $V_{S B}$ and $V_{t h}$ vary accordingly indicated by (8). Therefore, driving current of the uploading transistor can be tuned through body voltage



Fig. 18: The location of body-biasing sensing circuit in STTRAM sub-array.

variations. For example, if the data cell stores ' 0 ', i.e., the MTJ has a low resistance, $I_{d a t a 0}$ will be larger than $I_{r e f}$. Consequently, the voltage drop over uploading transistors in data branch (i.e., PDD and PLD) is larger than those in reference branch (i.e., PDR0 and PLR0 or PDR1 and PLR1). Then, $V_{d a t a 0}$ will be lower than $V_{\text {ref }}$. As a result, sourcebody voltage of PLD would be smaller than that of PLR0/1. So driving current of PLR increases while those of PDR0 and PDR1 decreases. Consequently, the difference between data sensing voltage and reference voltage will be enlarged further, and read margin can be improved. The case when sensing data ' 1 ' can be analyzed similarly. The detailed experimental results are presented in the next subsection.

Fig.18 shows how our proposed sensing circuit can be integrated in the STT-RAM sub-array. As shown in the figure, due to sensing amplifiers are shared among multiple column cells and our proposed technique only changes the bodybiasing connections to uploading transistors in sensing circuit, the incurred area overhead is negligible.

\section{B. Read reliability improvement by our proposed body-biasing based sensing circuit design}

To evaluate read margin and reliability enhancement, we use 1T-1MTJ memory cell structure mentioned above in the following electrical simulations. Fig. 19 plots waveforms of $V_{\text {data }}$ and $V_{\text {ref }}$ during reading ' 0 ' and ' 1 ' when using our body-biasing technique compared to the case without using it at $25^{\circ} \mathrm{C}$ in $45 \mathrm{~nm}$ technology node. It shows that voltage difference is magnified dramatically by body-biasing. The read margin increases by nearly $3 \mathrm{X}$, for both reading ' 0 ' and reading ' 1 ', which is helpful to reduce read error rate for the second sensing stage. The experimental results on $11 \mathrm{~nm}$ 1T1MTJ STT-RAM also show the similar trend. Then, we plot the read margin comparisons at different temperatures in Fig. 20. As shown in the figure, sensing margins of our method are significantly higher than those of [8] over the temperature range from $-20^{\circ} \mathrm{C}$ to $100^{\circ} \mathrm{C}$. Note that read margin of reading ' 1 ' is much larger than that of reading ' 0 ' by body-biasing technique. The reason is that when reading ' 0 ', the reference 


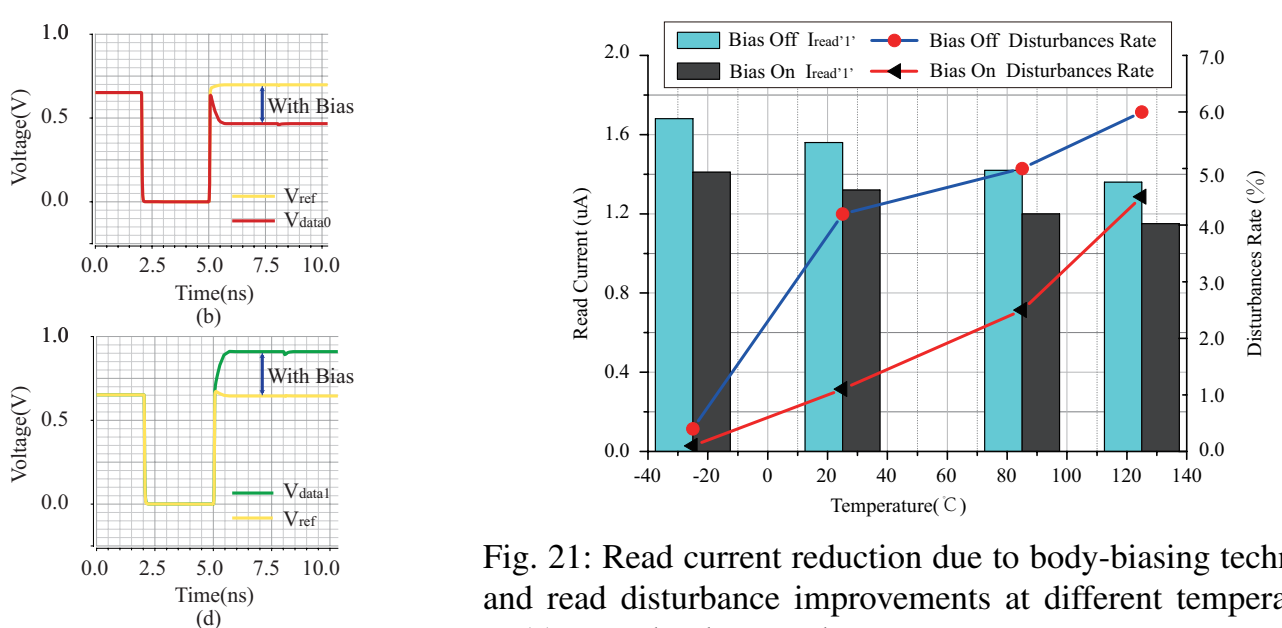

Fig. 21: Read current reduction due to body-biasing technique and read disturbance improvements at different temperatures at $11 \mathrm{~nm}$ technology node.

Fig. 19: Sensing signal waveforms when reading ' 0 ' and reading ' 1 '. (a) The sensing ' 0 ' voltage waveforms when using the circuit proposed by [8]. (b) The sensing ' 0 ' voltage waveforms when using our proposed technique. (c) The sensing ' 1 ' voltage waveforms when using the circuit proposed by [8]. (d) The sensing ' 1 ' voltage waveforms when using our proposed technique.



Fig. 20: Read margin improvements by body-biasing technique at different temperatures when read ' 0 ' and read ' 1 ' respectively.

voltage is higher than data voltage, and approaches nominal body voltage of PMOS transistor more closely. Therefore the tuning head room is reduced for reading ' 1 ' case. Another interesting point is that read margin improvement shrinks with temperature increasing. This is attributed to the fact that MOS transistor carrier mobility deteriorates significantly at high temperature making body-biasing less effective on tuning transistor driving currents. However, on average, our proposed sensing scheme can enlarge read margin by $2.47 \mathrm{X}$ when reading ' 0 ' and $3.15 \mathrm{X}$ when reading ' 1 '. The enhanced read margin guarantees that the data can be sensed more reliably and read error rate can be reduced accordingly. Then, we evaluate the read error rate when using our proposed sensing circuit at different temperatures in $45 \mathrm{~nm}$ technology node. We obtain the simulation result through 1000 Monte Carlo simulations with $4 \%$ MOS transistor and MTJ size variations, and there is no read error rate any more due to enlarged read margin until $100^{\circ} \mathrm{C}$ where body-biasing technique can not tune the driving ability of transistor effectively.

As mentioned in Section IV.D, read disturbance becomes significant when MTJ size shrinks from $40 \mathrm{~nm}$ to $1 \mathrm{Xnm}$. Since body-biasing technique can be helpful on enlarging read margin, we can reduce the read current with the same read margin constraint. Fig. 21 depicts the read current reduction due to body-biasing technique and read disturbance improvement at different temperatures on $11 \mathrm{~nm}$ MTJ technology node. We can observe that read current of body-biasing sensing circuit can reduce read current by $20 \%$ on average with the same read margin of original sensing circuit design ${ }^{2}$. Therefore, read disturbance can be alleviated due to reduced read current. Fig. 21 also shows read disturbance reductions at different temperatures when compared to those of the original sensing circuit. It clearly indicates that read error rate due to read disturbance can be reduced effectively. On average, the read disturbance error rate can be reduced by $55.6 \%$ when using our proposed body-biasing technique.

\section{RELATED WORK}

Since STT-RAM has many advantages to make it a promising candidate for future memory technologies, it has attracted much attentions from both academia and industry. The STTRAM research can be divided into different levels. At device level, a compact model of MTJ is essential for upper level simulation and analyses. Sun investigated spin current induced MTJ switching and described switching behavior by LandauLifshitz-Gilbert equation accurately making it a base for following compact model development [38]. Faber et al. proposed a compact model of MTJ for in-plane STT-RAM, which can capture both static and dynamic characteristics [6]. Guo et al. investigated MTJ switching induced by Spin Transfer torque effect and built a SPICE compact model for STTRAM simulation [27]. Shang et al. developed an SPICElike simulation tool integrating MTJ switching model. This

\footnotetext{
${ }^{2}$ The relative small current reduction of read current compared to read margin amplification is that the resistance of $11 \mathrm{~nm}$ MTJ is much larger than $45 \mathrm{~nm}$ MTJ case. The large voltage difference only converted to much smaller read current variation.
} 
tool models MTJ switching behavior using electrical devices such as current source, resistance, capacitance, etc, and it supports hybrid CMOS/MTJ circuit simulation. However, due to the complex equivalent circuit, simulation overhead is nonnegligible [39]. Zhao et al. developed a model for thermally assisted MTJ model [28]. Zhang et al. developed a compact model for perpendicular anisotropy MTJ using Verilog-A at 40nm technology node [28]. With this model, authors explored tradeoffs involved in designing non-volatile flip-flops. Based on this work, Wang et al. enhanced the compact model to capture MTJ stochastic switching property and evaluate $\mathrm{MgO}$ barrier breakdown effect on MTJ reliability [29]. Fong et al. developed a verilog-A MTJ model based on LLG equation and Green function method which can model both in-plane and perpendicular MTJ switching procedure accurately [30]. Unfortunately, none of above models can capture temperature effect on MTJ and STT-RAM access behavior effectively. However, as the on-chip power consumption and temperature increases sharply, it is imperative to investigate temperature effect on STT-RAM access performance, power consumption and reliability to derive an optimal design across the whole operation temperature range of the specified STT-RAM product.

Shang et al. investigated temperature effect on spin polarized tunneling of MTJ built with different ferromagnetic materials [18]. The authors observed that both junction resistance and magnetoresistance decreases with temperature increases. Hagler et al. explored temperature dependence of MTJ with $\mathrm{Al}_{2} \mathrm{O}_{3}$ barrier experimentally [19], which also revealed that MTJ magnetoreistance reduction as temperature increased while TMR value showed a decreasing trend. Lee et al. emphasized importance of thermal aware design of STTRAM and illustrated temperature effect on MTJ resistance and switching current [17]. A 45nm prototype STT-RAM from Lin et al. also demonstrated that temperature has significant impact on MTJ switching current and anti-parallel resistance [21]. However, these research efforts only focus on the device level and lack of temperature effect evaluation on circuit and architecture level. Considering the sensing circuit design, J. Kim proposed a degeneration loading scheme to mitigate process variation impact on STT-RAM sensing circuit [40]. Based on this sensing structure, they improved its sensing reliability further by self body-biasing technique in [40]. However, uploading transistors on both data and reference paths have the same body potential and can not benefit from dynamically changed reference voltage level to achieve larger read margin. Additionally, they did not analyze temperature variations on MTJ access behavior in detail. Bi et. al. investigated the temperature dependence of in-plane STT-RAM cell, which is the most relevant literature to our work. However, they only considered in-plane STT-RAM [41]. To fill the gap between device and upper level study concerning thermal effect, we build a thermal-aware MTJ model for hybrid MTJ/CMOS electrical simulation to capture temperature factors during MTJ switching and use it for circuit and architecture level analyses of STT-RAM.

\section{CONCLUSION \& FUTURE WORK}

As the integration density of conventional CMOS transistor sharply increases, power consumption of memory system makes it a road block for high performance exascale computing. Several emerging non-volatile memory technologies are proposed to replace traditional SRAM or DRAM to achieve low power and memory consistency. Among them, STT-RAM is a promising candidate as on-chip cache or main memory due to its high integration density, high access speed, and compatibility with CMOS fabrication process. However, MTJ as the core component of STT-RAM is sensitive to temperature fluctuations. Therefore, it is imperative to investigate performance and reliability of STT-RAM taking thermal factor into account. In the article, we build a thermal model which can capture thermal behavior of MTJ and support hybrid MTJ/CMOS electrical simulations. Based on the model, we evaluate thermal effect on 1T1MTJ memory cell from performance, latency and reliability perspectives. We also predict the temperature effect at more advanced $1 \mathrm{Xnm}$ technology node to analyze scalability of STT-RAM technology. After that, we propose a body-biasing sensing scheme to improve read margin and read reliability over the whole working temperature range. Experimental results show that our proposed technique can improve read reliability effectively. Additionally, with the same read margin constraint, it can reduce read disturbance significantly due to smaller sensing current at more advanced technology node. Our future work will be investigation of design techniques that ensure sensing reliability at very high temperature $\left(>100^{\circ} \mathrm{C}\right)$ when it is difficult to adjust driving abilities of transistors by body-biasing.

\section{REFERENCES}

[1] N. S. Kim, T. Austin, D. Baauw, T. Mudge, K. Flautner, J. S. Hu, M. J. Irwin, M. Kandemir, and V. Narayanan, "Leakage current: Moore's law meets static power," IEEE Computer, vol. 36, no. 12, pp. 68-75, 2003.

[2] M. B. Taylor, "A landscape of the new dark silicon design regime," IEEE Micro, vol. 33, no. 5, pp. 8-19, 2013.

[3] H. Esmaeilzadeh, E. Blem, R. S. Amant, K. Sankaralingam, and D. Burger, "Dark silicon and the end of multicore scaling," in Proc. IEEE/ACM International Symposium on Computer Architecture (ISCA'11), San Jose, USA, Jun.4-8 2011, pp. 365-376.

[4] J. Li, P. Ndai, A. Goel, S. Salahuddin, and K. Roy, "Design paradigm for robust spin-torque transfer magnetic ram (STT MRAM) from circuit/architecture perspective," IEEE Trans. VLSI Syst., vol. 18, no. 12, pp. 1710-1723, 2010.

[5] W. Zhao and G. Prenat, Spintronics-based Computing. Springer: Berlin, Germany, 2015.

[6] L.-B. Faber, W. Zhao, J.-O. Klein, T. Devolder, and C. Chappert, "Dynamic compact model of spin-transfer torque based magnetic tunnel junction (MTJ)," in Proc. IEEE Design \& Technology of Integrated Systems in Nanoscale Era (DTIS'09), Cairo, Egypt, Apr.6-9 2009, pp. 130-135.

[7] L. Su, Y. Zhang, J.-O. Klein, Y. Zhang, A. Bournel, A. Fert, and W. Zhao, "Current-limiting challenges for all-spin logic devices," Scientific Reports, vol. 5, p. 14905, 2015.

[8] J. Kim, K. Ryu, S. H. Kang, and S.-O. Jung, "A novel sensing circuit for deep submicron spin transfer torque MRAM (STT-MRAM)," IEEE Trans. VLSI Syst., vol. 20, no. 1, pp. 181-186, 2012.

[9] H. Noguchi, K. Ikegami, K. Kushida, K. Abe, S. Itai, S. Takaya, N. Shimomura, J. Ito, A. Kawasumi, H. Hara, and S. Fujita, "A 3.3nsaccess-time $71.2 \mu \mathrm{w} / \mathrm{mhz} 1 \mathrm{mb}$ embedded STT-MRAM using physically eliminated read-disturb scheme and normally-off memory architecture," in Proc. IEEE International Solid- State Circuits Conference (ISSCC'15), San Francisco, USA, Feb.22-26 2015, pp. 136-138. 
[10] Z. Sun, X. Bi, H. Li, W.-F. Wong, and X. Zhu, "STT-RAM cache hierarchy with multiretention MTJ designs," IEEE Trans. VLSI Syst., vol. 22, no. 6, pp. 1281-1293, 2014.

[11] R. Wang, L. Jiang, Y. Zhang, L. Wang, and J. Yang, "Selective restore: an energy efficient read disturbance mitigation scheme for future STTMRAM," in Proc. IEEE/ACM Design Automation Conference (DAC'15), San Francisco, USA, Jun.7-11 2015, pp. 1-6.

[12] Z. Pajouhi, X. Fong, and K. Roy, "Device/circuit/architecture co-design of reliable STT-MRAM," in Proc. IEEE/ACM Conference on Design, Automation and Test in Europe (DATE'15), Grenoble, France, Mar.9-13 2015, pp. 1437-1442.

[13] Y. Lu, T. Zhong, W. Hsu, S. Kim, X. Lu, J. J. Kan, C. Park, W. C. Chen, X. Li, X. Zhu, P. Wang, M. Gottwald, J. Fatehi, L. Seward, J. P. Kim, N. Yu, G. Jan, J. Haq, S. Le, Y. J. Wang, L. Thomas, J. Zhu, H. Liu, Y. J. Lee, R. Y. Tong, K. Pi, D. Shen, R. He, Z. Teng, V. Lam, R. Annapragada, T. Torng, P.-K. Wang, and S. H. Kang, "Fully functional perpendicular STT-MRAM macro embedded in $40 \mathrm{~nm}$ logic for energy-efficient IOT applications," in Proc. IEEE International Electron Devices Meeting (IEDM'15), Washington, USA, Dec. 7-9, 2015, pp. 26.1.1-26.1.4.

[14] W. Kang, L. Zhang, J.-O. Klein, Y. Zhang, D. Ravelosona, and W. Zhao, "Reconfigurable codesign of STT-MRAM under process variations in deeply scaled technology," IEEE Trans. Electron Devices, vol. 62, no. 6, pp. 1769-1777, 2015.

[15] A. Vatankhahghadim, W. Song, and A. Sheikholeslami, "A variationtolerant MRAM-backed-SRAM cell for a nonvolatile dynamically reconfigurable FPGA," IEEE Trans. Circuits Syst. II, vol. 62, no. 6, pp. 573-577, 2015.

[16] J. Li, H. Liu, S. Salahuddin, and K. Roy, "Variation-tolerant spintorque transfer (STT) MRAM array for yield enhancement," in Proc. IEEE Custom Integrated Circuits Conference (CICC'08), San Jose, CA, Sep.21-24 2008, pp. 193-196.

[17] K. Lee and S. H. Kang, "Design consideration of magnetic tunnel junctions for reliable high-temperature operation of STT-MRAM," IEEE Trans. Magn., vol. 46, no. 6, pp. 1537-1540, 2010.

[18] C. H. Shang, J. Nowak, R. Jansen, and J. S. Moodera, "Temperature dependence of magnetoresistance and surface magnetization in ferromagnetic tunnel junctions," Physical Review B, vol. 58, pp. R2917R2920, 1998.

[19] T. Hagler, R. Kinder, and G. Bayreuther, "Temperature dependence of tunnel magnetoresistance," Journal of Applied Physics, vol. 89, no. 11, p. 7570, 2001

[20] T. Kishi, H. Yoda, T. Kai, T. Nagase, E. Kitagawa, M. Yoshikawa, K. Nishiyama, T. Daibou, M. Nagamine, M. Amano, S. Takahashi, M. Nakayama, N. Shimomura, H. Aikawa, S. Ikegawa, S. Yuasa, K. Yakushiji, H. Kubota, A. Fukushima, M. Oogane, T. Miyazaki, and K. Ando, "Lower-current and fast switching of a perpendicular TMR for high speed and high density spin-transfer-torque MRAM,' in Proc. IEEE International Electron Devices Meeting (IEDM'08), San Francisco, USA, Dec.15-17 2008, pp. 1-4.

[21] C. J. Lin, S. H. Kang, Y. J. Wang, K. Lee, X. Zhu, W. C. Chen, X. Li, W. N. Hsu, Y. C. Kao, M. T. Liu, W. C. Chen, Y. Lin, M. Nowak, N. Yu, and L. Tran, " $45 \mathrm{~nm}$ low power CMOS logic compatible embedded STT MRAM utilizing a reverse-connection 1T/1MTJ cell," in Proc. IEEE International Electron Devices Meeting (IEDM'09), Baltimore, USA, Dec.7-9 2009, pp. 256-259.

[22] S. Ikeda, H. Sato, H. Honjo, E. C. I. Enobio, S. Ishikawa, M. Yamanouchi, S. Fukami, S. Kanai, F. Matsukura, T. Endoh, and H. Ohno, "Perpendicular-anisotropy $\mathrm{CoFeB}-\mathrm{MgO}$ based magnetic tunnel junctions scaling down to 1x nm," in Proc. IEEE Electron Devices Meeting (IEDM'14), San Francisco, USA, Dec.15-17 2014, pp. 33.2.1-33.2.4.

[23] G. Jeong, C. Wooyoung, S. Ahn, J. Hongsik, G. Koh, H. Youngnam, and K. Kim, "A 0.24- $\mu$ m 2.0-v 1T1MTJ 16-kb nonvolatile magnetoresistance RAM with self-reference sensing scheme," IEEE J. Solid-State Circuits, vol. 38, no. 11, pp. 1906-1910, 2003.

[24] T. W. Andre, J. J. Nahas, C. K. Subramanian, B. J. Garni, H. S. Lin, A. Omair, and W. L. Martino, "A 4-Mb 0.18- $\mu m$ 1T1MTJ toggle MRAM with balanced three input sensing scheme and locally mirrored unidirectional write drivers," IEEE J. Solid-State Circuits, vol. 40, no. 1 , pp. 301-309, 2005.

[25] K. Skadron, M. R. Stan, W. Huang, V. Sivakumar, S. Karthik, and D. Tarjan, "Temperature-aware microarchitecture," in Proc. IEEE/ACM International Symposium on Computer Architecture (ISCA'03), San Diego, USA, Jun.9-11 2003, pp. 2- 13.

[26] A. Raychowdhury, D. Somasekhar, T. Karnik, and V. De, "Design space and scalability exploration of 1T-1STT MTJ memory arrays in the presence of variability and disturbances," in Proc. IEEE International
Electron Devices Meeting (IEDM'09), Baltimore, USA, Dec. 7-9 2009, pp. $1-4$.

[27] W. Guo, G. Prenat, V. Javerliac, M. E. Baraji, N. de Mestier, and C. . D. B. Baraduc, "Spice modelling of magnetic tunnel junctions written by spin-transfer torque," Journal of Physics D: Applied Physics, vol. 43, p. $215001,2010$.

[28] W. Zhao, J. Duval, J. O. Klein, and C. Chappert, "A compact model for magnetic tunnel junction (MTJ) switched by thermally assisted spin transfer torque (TAS + STT)," Nanoscale Research Letters, vol. 6, no. 1 , p. 368, 2011.

[29] Y. Wang, Y. Zhang, E. Y. Deng, J. O. Klein, L. A. B. Naviner, and W. S. Zhao, "Compact model of magnetic tunnel junction with stochastic spin transfer torque switching for reliability analyses," Microelectronics Reliability, vol. 54, no. 9-10, pp. 1774-1778, 2014

[30] X. Fong, S. H. Choday, P. Georgios, C. Augustine, and K. Roy, "Purdue nanoelectronics research laboratory magnetic tunnel junction model," Oct 2014. [Online]. Available: https://nanohub.org

[31] Y. Zhang, W. Zhao, Y. Lakys, J. O. Klein, J.-V. Kim, D. Ravelosona, and C. Chappert, "Compact modeling of perpendicular-anisotropy $\mathrm{CoFeB} / \mathrm{MgO}$ magnetic tunnel junctions," IEEE Trans. Electron Devices, vol. 59, no. 3, pp. 819-826, 2012.

[32] S. Ikeda, K. Miura, H. Yamamoto, K. Mizunuma, H. D. Gan, M. Endo, S. Kanai, J. Hayakawa, F. Matsukura, and H. Ohno, "A perpendicularanisotropy $\mathrm{CoFeBCMgO}$ magnetic tunnel junction," Nature Materials, vol. 9, no. 9, pp. 721-724, 2010.

[33] T. L. Gilbert, "Classics in magnetics a phenomenological theory of damping in ferromagnetic materials," IEEE Trans. Magn., vol. 40, no. 6, pp. 3443-3449, 2004

[34] W. Zhao, C. Chappert, V. Javerliac, and J. P. Noziere, "High speed, high stability and low power sensing amplifier for MTJ/CMOS hybrid logic circuits," IEEE Trans. Magn., vol. 45, no. 10, pp. 3784-3787, 2009.

[35] Predictive technology model. [Online]. Available: http://ptm.asu.edu

[36] Cadence. Spectre circuit simulator. [Online]. Available: http://www.cadence.com

[37] X. Dong, X. Wu, G. Sun, Y. Xie, H. Li, and Y. Chen, "Circuit and microarchitecture evaluation of 3D stacking magnetic ram MRAM as a universal memory replacement," in Proc. IEEE/ACM Design Automation Conference (DAC'08), Austin, USA, Jun.5-9 2008, pp. 554-559.

[38] J. Z. Sun, "Spin-current interaction with a monodomain magnetic body: A model study," Physical Review B, vol. 62, no. 1, p. 570, 2000.

[39] Y. Shang, W. Fei, and H. Yu, "Fast simulation of hybrid CMOS and STTMTJ circuits with identified internal state variables," in Proc. IEEE/ACM Asia and South Pacific Design Automation Conference (ASP-DAC'12), Sydney, Australia, Jan.30-Feb. 2 2012, pp. 529 - 534.

[40] J. Kim, K. Ryu, J. P. Kim, S. H. Kang, and S.-O. Jung, "STT-MRAM sensing circuit with self-body biasing in deep submicron technologies," IEEE Trans. VLSI Syst., vol. 22, no. 7, pp. 1630-1634, 2014.

[41] X. Bi, H. Li, and X. Wang, "STT-RAM cell design considering CMOS and MTJ temperature dependence," IEEE Trans. Magn., vol. 48, no. 11, pp. 3821-3824, 2012.

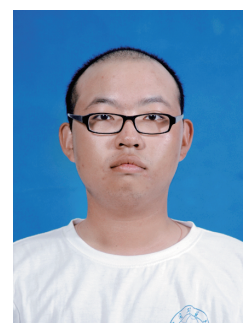

Bi Wu received his B.S. and M.S. degree from China University of Mining and Technology, Xuzhou, China, and Beihang University, Beijing, China respectively. He is persuing Ph.D. degree of Electrical Engineering at Beihang University. His research interests include circuit level design and optimization of STT-RAM, STT-RAM reliability analysis and improvement, etc. 


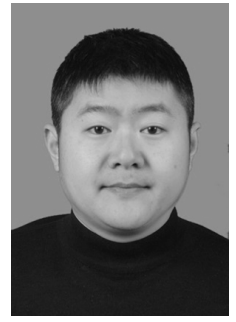

Yuanqing Cheng (S'11, M'13) received his Ph.D. degree from the Key Laboratory of Computer System and Architecture, Institute of Computing Technology, Chinese Academy of Sciences, Beijing, China. After spending one year post-doc study at LIRMM, CNRS, France, he joined Beihang University, China as an assistant professor. His research interests include VLSI design for 3D integrated circuits considering thermal and defect issues, as well as spintronics computing system architecture design. $\mathrm{He}$ is currently an IEEE member and ACM member.

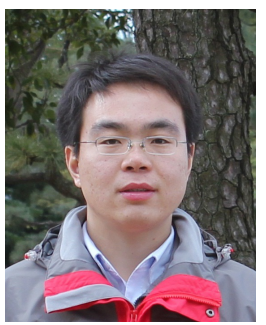

Jianlei Yang (S'12) received the B.S. degree in microelectronics from Xidian University, Xi' an, China, in 2009, and the Ph.D. degree in computer science and technology with Tsinghua University, Beijing, China, in 2014. Dr. Yang is currently a postdoctoral researcher with the Department of Electrical and Computer Engineering, University of Pittsburgh, Pittsburgh, Pennsylvania, United States. From 2013 to 2014, he was a research intern at Intel Labs China, Intel Corporation. His current research interests include numerical algorithms for VLSI power grid analysis and verification, spintronics and neuromorphic computing. Dr. Yang was the recipient of the first place on TAU Power Grid Simulation Contest in 2011, and the second place on TAU Power Grid Transient Simulation Contest in 2012. He was a recipient of IEEE ICCD Best Paper Award in 2013, and ACM GLSVLSI Best Paper Nomination in 2015.



Aida Todri-Sanial (M'03) received the B.S. degree in electrical engineering from Bradley University, IL in 2001, the M.S. degree in electrical engineering from Long Beach State University, CA, in 2003 and Ph.D. degree in electrical and computer engineering from the University of California, Santa Barbara, in 2009. She is currently a research scientist for French National Center of Scientific Research (CNRS) attached to Laboratoire dInformatique de Robotique et de Microlectronique de Montpellier (LIRMM). Previously she was an RD Engineer for Fermi National Accelerator Laboratory, IL where she was the recipient of John Bardeen Fellow in Engineering in 2009. She has also held visiting positions at Mentor Graphics, Cadence Design Systems, STMicroelectronics and IBM TJ Watson Research Center.

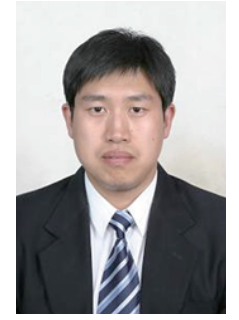

Weisheng Zhao (M'06, SM'14) received the Ph.D. degree in physics from the University of Paris-Sud, France, in 2007. From 2004 to 2008, he investigated Spintronic devices based logic circuits and designed a prototype for hybrid Spintronic/CMOS $(90 \mathrm{~nm})$ chip in cooperation with STMicroelectronics. Since 2009, he joined the CNRS as a tenured research scientist and his interest includes the hybrid integration of nano-devices with CMOS circuit and new non-volatile memory $(40 \mathrm{~nm}$ technology node and below) like MRAM circuit and architecture design. He has authored or co-authored more than 150 scientific papers (e.g Advanced Material, Nature Communications, IEEE Transactions etc.); he is also the principal inventor of 4 international patents. From 2014, he becomes a youth 1000 plan distinguished professor in Beihang University, Beijing, China, and the associated editor for IEEE Transactions on Nanotechnology. 